\title{
NUEVOS HALLAZGOS \\ DE MICROBIOTHERIIDAE (MAMMALIA, MARSUPIALIA) EN LA FORMACIÓN PINTURAS (MIOCENO TEMPRANO, ARGENTINA)
}

\author{
LAURA CHORNOGUBSKY y ALEJANDRO G. KRAMARZ
}

Sección Paleontología Vertebrados, Museo Argentino de Ciencias Naturales "Bernardino Rivadavia”, Av. Ángel Gallardo 470, C1405DJR Buenos Aires, Argentina Consejo Nacional de Investigaciones Científicas y Técnicas (CONICET). lchorno@macn.gov.ar, agkramarz@macn.gov.ar

\begin{abstract}
Resumen. La Formación Pinturas (Mioceno temprano, noroeste de la provincia de Santa Cruz, Argentina) es una sucesión continental divisible en tres secuencias sobre la base de las principales superficies erosivas intraformacionales y los cambios litológicos. Los estudios previos concluyeron que las secuencias inferior y media tienen una edad absoluta mayor a 16,5 Ma y son portadoras de una asociación de mamíferos ("fauna Astrapotericulense") distinta y más antigua que la que tipifica la Edad Mamífero Santacrucense. Recientemente, la primera especie de un marsupial microbiotérido, Pachybiotherium illuminatum Goin, Tejedor, Abello y Martin, fue descripta para la secuencia media de esta unidad. En el presente trabajo se describen nuevos especímenes de microbiotéridos, representados por elementos dentarios, procedentes de la misma secuencia en tres localidades de la provincia de Santa Cruz: Cerro de los Monos, Portezuelo Sumich Norte y Portezuelo Sumich Sur. Estos ejemplares son referidos a Microbiotherium tehuelchum Ameghino, Microbiotherium divisum Ameghino y Microbiotherium cf. M. gallegosense Sinclair. Al igual que lo observado para otros mamíferos procedentes de estos niveles de la Formación Pinturas, la asociación de marsupiales microbiotéridos está constituida por una combinación de taxones previamente conocidos para la Edad Santacrucense, taxones conocidos para la Edad Colhuehuapense y un taxón exclusivamente conocido para estos niveles (Pachybiotherium illuminatum). Esta combinación refuerza la idea de una asociación "Pinturense", distinta y de antigüedad intermedia entre las edades mamífero Colhuehuapense y Santacrucense. Las cuatro especies de microbiotéridos representan la comunidad más diversa hasta ahora conocida para estos marsupiales, ya que se registran positivamente en una misma localidad y un mismo nivel estratigráfico (i.e., Portezuelo Sumich Sur).
\end{abstract}

Palabras clave. Microbiotheriidae. Marsupialia. Formación Pinturas. Mioceno temprano. Patagonia. América del Sur.

Abstract. MICROBIOTHERIIDAE (MAMMALIA, MARSUPIALIA) FROM THE PINTURAS FORMATION (EARLY MIOCENE, ARGENTINA). The Pinturas Formation (early Miocene, northwestern Santa Cruz Province, Argentina) is a continental succession that can be divided into three sequences based on its major intraformational erosive surfaces and lithological changes. Previous studies concluded that the lower and middle sequences have an absolute age of more than $16.5 \mathrm{Ma}$ and they bear a mammal assemblage ("Astrapothericulan fauna") different from and older than that typifying the Santacrucian Land Mammal Age. Recently, the first microbiotheriid marsupial species, Pachybiotherium illuminatum Goin, Tejedor, Abello, and Martin, was described from the middle sequence of this unit. In this contribution, new microbiotheriid specimens from the same sequence, represented by dental elements, are described from three localities of Santa Cruz Province: Cerro de los Monos, Portezuelo Sumich Norte, and Portezuelo Sumich Sur. These specimens are referred to Microbiotherium tehuelchum Ameghino, Microbiotherium divisum Ameghino, and Microbiotherium cf. M. gallegosense Sinclair. Similarly to what was observed for other mammals from these levels of the Pinturas Formation, the microbiotheriid association includes a combination of taxa previously known from the Santacrucian SALMA, others known from the Colhuehuapian SALMA, and a taxon recorded exclusively in these levels (Pachybiotherium illuminatum). This combination of taxa reinforces the idea of the "Pinturan assemblage" as distinct and intermediate in age between the Colhuehuapian and the Santacrucian. The four microbiotheriids represent the most diverse community so far known for this marsupial group as they positively occur in a single locality and stratigraphic level (i.e., Portezuelo Sumich Sur).

Key words. Microbiotheriidae. Marsupialia. Pinturas Formation. Early Miocene. Patagonia. South America.

Los Microbiotheriidae constituyen un grupo de marsupiales australidelfios de América del Sur (Szalay, 1982). Algunos autores proponen una posición basal al clado Australidelphia para este grupo (e.g., Szalay, 1982; Nilsson et al., 2010). Otros (e.g., Palma y Spotorno, 1999; Asher et al., 2004) incluyen a los Microbiotheriidae entre los Australidelphia, como grupo hermano de distintos marsupiales australianos.

El biocrón de los Microbiotheriidae se extiende desde el Paleoceno temprano (Marshall y de Muizon, 1988) has- ta el presente, con una única especie viviente (Dromiciops gliroides Thomas), cuya distribución está restringida a la ecorregión de los bosques templados valdivianos (Olson et al., 2001 fide Martin, 2010), principalmente en Chile, aunque también hay diversos registros en el oeste de las provincias de Neuquén, Río Negro y Chubut, en Argentina (Thomas, 1894; Martin, 2003, 2010). En el pasado, su distribución se extendió tan al norte como Colombia (Pachybiotherium minor Goin, 1995; Grupo Honda, Mioceno medio) y tan 
al sur como la Península Antártica (Marambiotherium glacialis Goin, Woodburne, Case, Vizcaíno y Reguero, 1999; Formación La Meseta, Eoceno). Sin embargo, la mayoría de los microbiotéridos fósiles se conocen principalmente para estratos del Mioceno temprano del centro y sur de Patagonia (géneros Microbiotherium Ameghino, 1887 y Pachybiotherium Ameghino, 1902; para una revisión de la familia, véase Marshall, 1982).

En el presente trabajo se dan a conocer nuevos materiales de marsupiales Microbiotheriidae, exhumados de niveles del Mioceno temprano de la Formación Pinturas, (provincia de Santa Cruz; Bown et al., 1988). Estos depósitos son portadores de una asociación de mamíferos fósiles a la que Ameghino (1906) denominó "fauna Astrapotericulense", sobre la que estableció su "Étage Astrapothericuléen" (Ameghino, 19001902, 1906). De acuerdo con Ameghino, la "fauna Astrapotericulense" es diferente y más antigua que la "Faune Notohippidéenne" (proveniente de la Formación Santa Cruz en la región

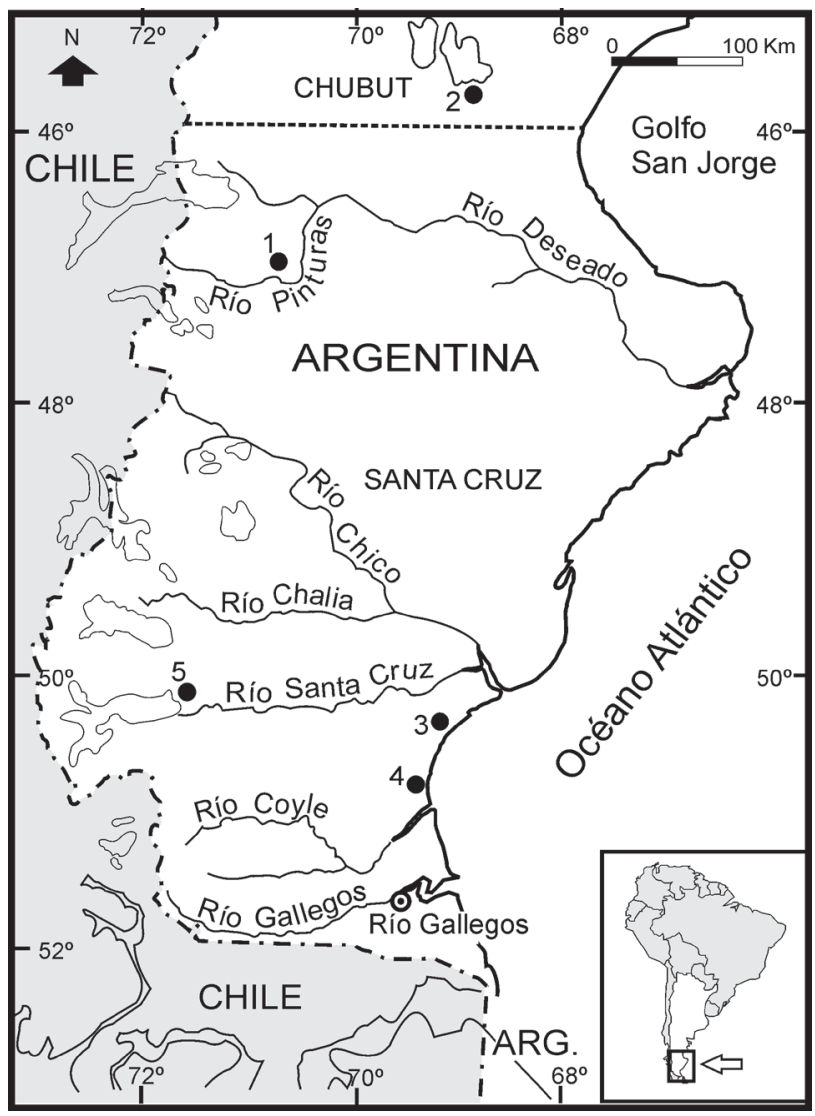

Figura 1. Mapa de Patagonia austral mostrando la ubicación del área del valle superior del río Pinturas (1), Barranca sur del Lago Colhué Huapi (2), Monte León (3), Monte Observación (4) y Karaiken (5). Modificado de Kramarz y Bellosi (2005)/ location map from southern Patagonia showing the area of the upper valley of the Pinturas river (1), Barranca south of Lago Colhué Huapi (2), Monte León (3), Monte Observación (4), and Karaiken (5). Modified from Kramarz and Bellosi (2005). de Karaiken; Fig. 1) y que la "Faune Santacruzéenne" (proveniente de las localidades costeras de la Formación Santa Cruz; Fig. 1), que tipifica la Edad Santacrucense (Simpson, 1940). Sin embargo, Wood y Patterson (1959) consideraron la "fauna Astrapotericulense" como una mera fauna local santacrucense, criterio luego seguido por autores posteriores (Pascual et al., 1965; Marshall et al., 1977, 1983). Las dataciones radiométricas (Fleagle et al., 1995) y los estudios bioestratigráficos más recientes (Barrio et al., 1984; Bown y Fleagle, 1993; Kramarz y Bellosi, 2005; Kramarz y Bond, 2005; Kramarz et al., 2010) concluyeron que al menos las secuencias inferior y media de la Formación Pinturas son portadoras de una asociación de mamíferos distinta y más antigua que aquella conocida para las típicas localidades de la Formación Santa Cruz.

El conjunto de mamíferos que constituye la "fauna Astrapotericulense" es muy diverso y rico en restos de notoungulados, litopternos, astrapoterios, xenartros, roedores, primates y marsupiales paucituberculados (Ameghino, 1900-1902, 1906; Barrio et al., 1984; Fleagle, 1990; Bown y Fleagle, 1993; Rae et al., 1996; Kramarz y Bellosi, 2005; Kramarz y Bond, 2005; González et al., 2006). Sin embargo, sólo recientemente fueron descriptos los primeros restos de marsupiales microbioterios de esta unidad (Goin et al., 2010). Los nuevos restos aquí descriptos permiten ampliar la diversidad conocida de estos marsupiales para la Formación Pinturas y discutir el significado biocronológico de estos hallazgos en relación a aquellos de otras asociaciones del Mioceno temprano de Patagonia.

Abreviaturas. Atad, ancho del talónido; Atrig, ancho del trigónido; Ltad, largo del talónido; Ltrig, largo del trigónido; MACN A, Museo Argentino de Ciencias Naturales, colección Ameghino; MACN Pv, Museo Argentino de Ciencias Naturales, Colección Nacional de Paleovertebrados; MEB, Microscopio Electrónico de Barrido; MLP, colección del Museo de La Plata. Mx, molar superior de locus x; mx, molar inferior de locus x; StX, cúspide estilar X.

\section{MARCO GEOLÓGICO Y ESTRATIGRÁFICO}

La Formación Pinturas es una sucesión de sedimentos predominantemente eólicos (mayormente tobáceos) de unos $100 \mathrm{~m}$ de espesor acumulados en las zonas periféricas elevadas del noroeste de la cuenca de Magallanes (Bown et al., 1988; Bown y Larriestra, 1990). Aflora principalmente en numerosas áreas aisladas en el valle superior del río Pinturas, en la zona precordillerana del noroeste de la provincia de Santa Cruz, Argentina (Fig. 1). Esta unidad presenta dos discordancias erosivas intraformacionales principales que definen tres secuen- 
cias (Fig. 2). La secuencia inferior ( $40 \mathrm{~m}$ ) está caracterizada por paleosuelos tufáceos muy maduros con trazas de nidos de insectos, pelitas piroclásticas con icnofósiles de roedores y lutitas carbonáceas con restos de peces (Bown y Larriestra, 1990; Bown y Laza, 1990; Genise y Bown, 1994; Kramarz y Bellosi, 2005). La secuencia media (30 m) está dominada por uno o dos niveles de psamitas epiclásticas correspondientes a paleodunas barjanoides con paleosuelos inmaduros (Bown y Larriestra, 1990). La secuencia superior está caracterizada por pelitas piroclásticas masivas con paleosuelos muy inmaduros con celdas fósiles de abejas (Bown y Larriestra, 1990; Genise y Bown, 1994). Los fósiles de vertebrados están principalmente concentrados en paleosuelos relacionados con incisiones fluviales o en paleosuelos pobre a moderadamente desarrollados de las tres secuencias (Bown y Fleagle, 1993).

Los ejemplares presentados en este trabajo proceden de la secuencia media de la Formación Pinturas, aflorante en las localidades Cerro de los Monos, Portezuelo Sumich Norte y Portezuelo Sumich Sur (Bown y Larriestra, 1990). En Cerro de los Monos, los restos fósiles ocurren directamente sobre el depósito de paleodunas desarrollado sobre la discordancia intraformacional que separa las secuencias inferior y media (Bown y Larriestra, 1990). En Portezuelo Sumich Norte los restos provienen de la base de un depósito fangolítico rosado y marrón que rellena el paleorrelieve correspondiente a la mencionada discordancia intraformacional. En Portezuelo Sumich Sur, los fósiles provienen de un depósito fangolítico arenoso gris ubicado también en la secuencia media, aunque aparentemente en una posición estratigráfica algo más alta que la de Cerro de los Monos (Bown y Larriestra, 1990). Las dataciones ${ }^{40} \mathrm{Ar} /{ }^{39} \mathrm{Ar}$ sugieren una edad absoluta mayor a 16,5 Ma para todos estos niveles (Fleagle et al., 1995).

\section{PALEONTOLOGÍA SISTEMÁTICA}

Infraclase Metatheria Huxley, 1880

Supercohorte MARsupiaLia Illiger, 1811

Cohorte Australidelphia Szalay, 1982

Orden Microbiotheria Ameghino, 1889

Familia Microbiotherimdae Ameghino, 1887

Género Microbiotherium Ameghino, 1887

Especie tipo. Microbiotherium patagonicum Ameghino, 1887.

Observaciones. Todos los ejemplares consignados más abajo al género Microbiotherium difieren de la especie tipo de Pachybiotherium (P. acclinum Ameghino, 1902) debido principalmente al mayor desarrollo del paracónido y del metacónido, este último de posición más mesial que en Pachybiotherium; la presencia de talónidos proporcionalmente más grandes, debido al mayor desarrollo del hipocónido; y el hipoconúlido más cercano al entocónido. En particular, además, difieren de Pachybiotherium illuminatum Goin, Tejedor, Abello y Martin, 2010 por la posición del hipoconúlido, más labial en este último, por su tamaño hasta cuatro veces menor y por presentar la rama mandibular proporcionalmente mucho más baja (Goin et al., 2011).

\section{Fm. Santa Cruz}

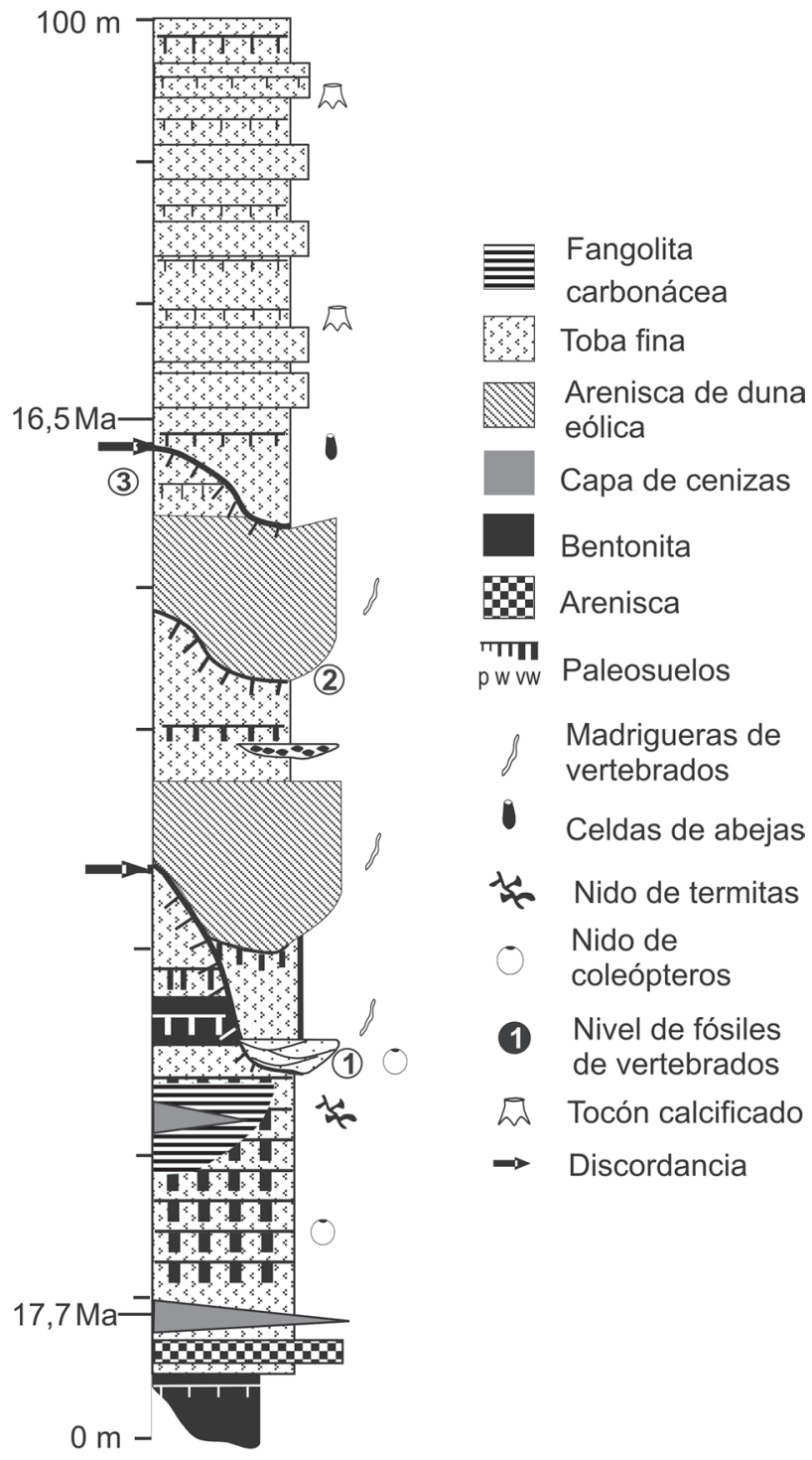

Grupo Bahía Laura

Figura 2. Perfil estratigráfico integrado de la Formación Pinturas/ integrated stratigraphic profile of the Pinturas Formation. 1, Portezuelo Sumich Norte; 2, Cerro de los Monos; 3, Portezuelo Sumich Sur. Modificado de/ modified from Kramarz y Bellosi (2005). 
Microbiotherium tehuelchum Ameghino, 1887

Figura 3.1

Neotipo. MLP 11-36, un fragmento de mandíbula derecha con los p2-m4 completos, proveniente de la Formación Santa Cruz, pero sin datos específicos de localidad (Marshall, 1982). Material referido. MACN Pv SC1172, un m2 derecho aislado; MACN Pv SC3622, un fragmento de dentario derecho con m2-3 (Fig. 3.1).

Procedencia geográfica y estratigráfica. Ambos ejemplares referidos provienen de la secuencia media de la Formación Pinturas. El ejemplar MACN Pv SC1172 proviene de la localidad Cerro de los Monos, mientras que el MACN Pv SC3622 proviene de Portezuelo Sumich Sur.

Observaciones. Los materiales asignados en el presente trabajo a $M$. tehuelchum son coincidentes con el neotipo designado por Marshall (1982) y otros ejemplares referidos por el mismo autor por la posición relativa de sus cúspides: paracónido de pequeño tamaño para el género, protocónido relativamente separado del metacónido, cíngulo anterobasal bien desarrollado, talónido más pequeño en el m3 que en el m2, con el hipocónido proyectado distalmente, entocónido proporcionalmente pequeño y la crístida oblicua más recta que en los molares de otras especies [e.g., $M$. divisum (Ameghino, 1902)]. La diferencia entre los ejemplares de la Formación Pinturas y los ejemplares asignados previamente a M. tehuelchum (Marshall, 1982) es que el tamaño de los ejemplares de la Formación Pinturas es algo mayor (Tab. 1). En particular, el ejemplar MACN Pv SC1172 es mayor que el MACN Pv SC3622 y sus cúspides son algo más globosas, siendo el MACN Pv SC3622 más similar en tamaño al neotipo de la especie.

Esta especie es la más abundante entre los microbioterios
(1)
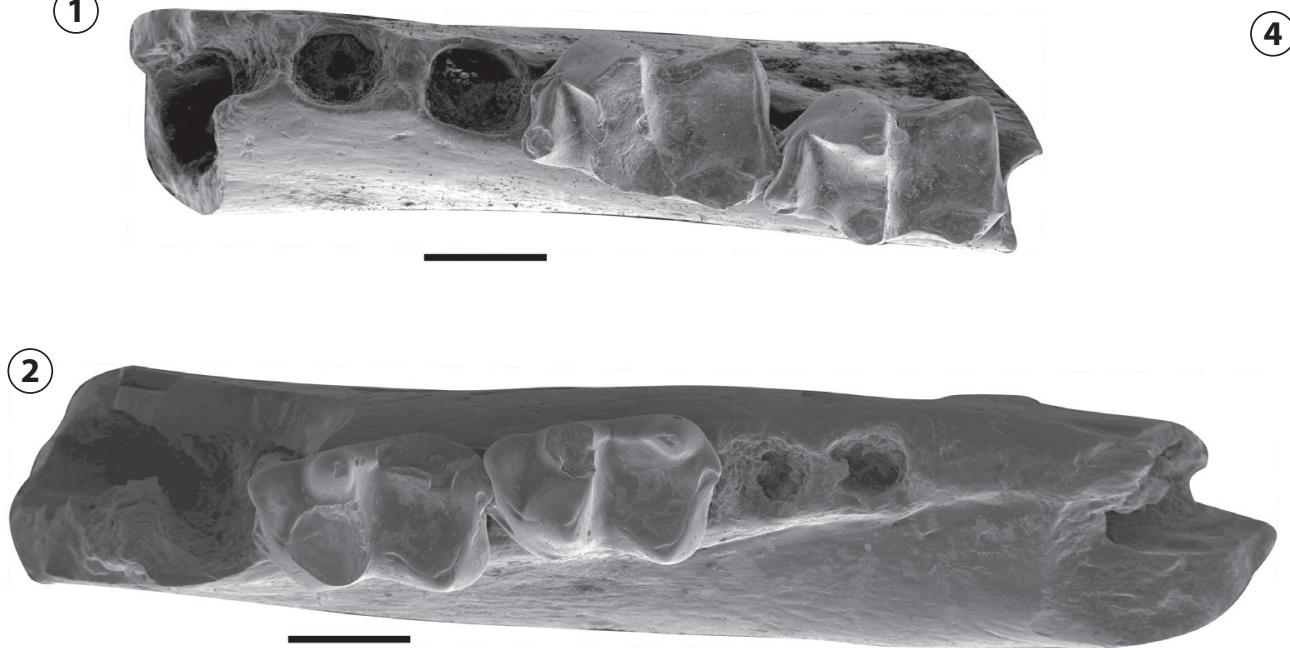

(3)

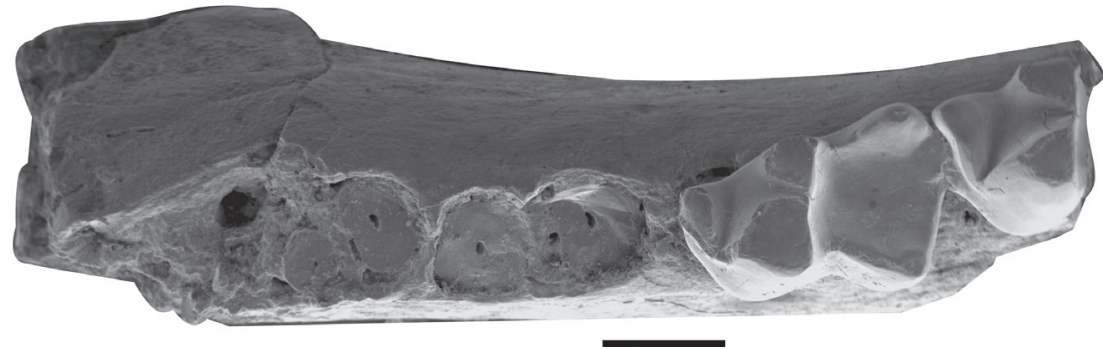

(4)

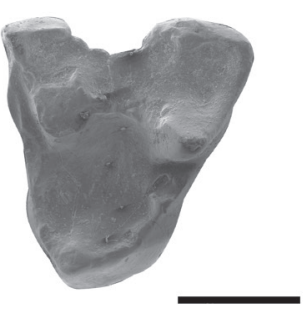

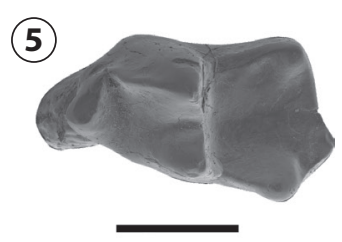

(6)

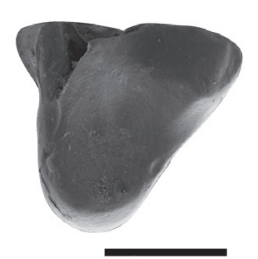

Figura 3. Fotografías de MEB de los microbiotéridos de la Formación Pinturas/ SEM photographs of the microbiotheriids from the Pinturas Formation. 1, Microbiotherium tehuelchum, MACN SC3622, fragmento mandibular derecho con los m2-3 en vista oclusal/ right mandibular fragment with m2-3, occlusal view. 2-4, Microbiotherium divisum, 2, MACN SC520, fragmento de dentario izquierdo con m2-3, vista oclusal/ left dentary fragment with $\mathrm{m} 2-3$, occlusal view; 3, MACN SC550, fragmento de dentario izquierdo con $\mathrm{m} 1$ y trigónido del $\mathrm{m} 2$, vista oclusal/ left dentary fragment with $m 1$ and trigonid of m2, occlusal view; 4, MACN SC3648, M2 izquierdo, vista oclusal/ left M2, occlusal view; 5, Microbiotherium cf. M. gallegosense, MACN $\mathrm{SC} 3645, \mathrm{~m} 1$ derecho, vista oclusal/ right m1, occlusal view; 6, Microbiotheriidae indet., MACN SC3648C, M2 izquierdo, vista oclusal/ left M2, occlusal view. Escala gráfica/scale bar $=1 \mathrm{~mm}$. 
conocidos para las localidades portadoras de fauna santacrucense de la Formación Santa Cruz y es la única especie de microbioterio reconocida para la Formación Río Frías en el sur de Chile (Marshall, 1990).

\section{Microbiotherium divisum (Ameghino, 1902)}

Figuras 3.2-4

Holotipo. MACN A 52-374, un fragmento de mandíbula derecha con el $\mathrm{m} 3$ roto en el ápice del metacónido y en parte del talónido, presuntamente proveniente del Miembro Colhué Huapi de la Formación Sarmiento, aflorante en la Barranca Sur del lago Colhué Huapi (también conocida como Gran Barranca).

Material referido. MACN Pv SC277, un fragmento de dentario izquierdo con los m2-4; MACN Pv SC520, un fragmento de dentario izquierdo con los m2-3 (Fig. 3.2); MACN Pv SC550, un fragmento de dentario izquierdo con el $\mathrm{m} 1$ completo y el trigónido del m2 (Fig. 3.3); MACN Pv SC3619B, un fragmento de dentario izquierdo con el m3 roto; MACN Pv SC3627, un fragmento de dentario izquierdo con los m3-4; MACN Pv SC3638, un fragmento de dentario derecho con el m4; MACN Pv SC3641, un fragmento de dentario derecho con el $\mathrm{m} 3$ completo y el trigónido del m4; MACN Pv SC3648, un M2 izquierdo aislado (Fig. 3.4).
Procedencia geográfica y estratigráfica. Todos los ejemplares provienen de la secuencia media de la Formación Pinturas. El ejemplar MACN Pv SC550 proviene de Cerro de los Monos. MACN Pv SC520 proviene de Portezuelo Sumich Norte. Los restantes ejemplares fueron hallados en Portezuelo Sumich Sur.

Medidas. Largo del M2: $2 \mathrm{~mm}$; ancho del M2: 1,72 $\mathrm{mm}$. Las medidas de los molares inferiores se indican en la Tabla 1. Observaciones. Esta especie constituye la única del género presente en estratos de la Edad Colhuehuapense. Originalmente asignada al género monotípico Oligobiotherium (Ameghino, 1902), fue reasignada con dudas a Microbiotherium por Pascual y Odreman Rivas (1971) y luego por Marshall (1982). Su característico protocónido, cónico y alto, y el gran desarrollo del hipocónido (dando como resultado un talónido proporcionalmente más grande que en otras especies), así como también la presencia de una crístida oblicua menos recta, hacen a esta especie claramente distinguible del resto. La presencia de estos caracteres en los materiales aquí presentados permite referirlos a esta especie. Estos caracteres pueden observarse claramente en los ejemplares figurados (Figs. 3.2-3), que se encuentran en vistas levemente distintas (Fig. 3.2, ejemplar en vista oclusal a oclusodistal; Fig. 3.3, ejemplar en vista oclusal a oclusomedial).

TABLA 1 - Medidas de los molares inferiores (en $\mathrm{mm}$ ) de Microbiotherium spp. de la Formación Pinturas/measurements of the lower molars of Microbiotherium spp. from the Pinturas Formation.

\begin{tabular}{|c|c|c|c|c|c|c|c|c|c|c|c|c|c|c|c|c|}
\hline \multirow[t]{2}{*}{$N^{\circ}$} & \multicolumn{4}{|c|}{$m 1$} & \multicolumn{4}{|c|}{$m 2$} & \multicolumn{4}{|c|}{$m 3$} & \multicolumn{4}{|c|}{$m 4$} \\
\hline & Ltrig & Ltad & Atrig & Atad & Ltrig & Ltad & Atrig & Atad & Ltrig & Ltad & Atrig & Atad & Ltrig & Ltad & Atrig & Atad \\
\hline \multicolumn{17}{|c|}{ M. tehuelchum } \\
\hline SC1172 & & & & & 1,10 & 1,27 & 1,23 & 1,50 & & & & & & & & \\
\hline SC3622 & & & & & $1,00^{*}$ & $1,07^{*}$ & $1,03^{*}$ & 1,27 & 0,97 & 1,07 & 1,10 & 1,13 & & & & \\
\hline \multicolumn{17}{|c|}{ M. divisum } \\
\hline SC277 & & & & & 1,07 & 1,33 & 1,23 & 1,50 & 1,17 & 1,10 & 1,17 & 1,37 & 0,93 & 0,60 & 0,93 & 0,60 \\
\hline SC520 & & & & & 1,07 & 0,97 & $1,07^{*}$ & $1,33^{*}$ & 0,97 & 1,03 & $1,07^{*}$ & 1,27 & & & & \\
\hline SC550 & $1,00^{*}$ & $1,00^{*}$ & $1,07^{*}$ & 1,17 & 1,03 & & 1,17 & & & & & & & & & \\
\hline SC3627 & & & & & & & & & 1,00 & 1,00 & 1,1 & 1,17 & 0,83 & 0,73 & 0,93 & 0,63 \\
\hline SC3641 & & & & & & & & & $0,93^{*}$ & 1,17 & $1,20^{*}$ & 1,30 & 0,90 & & 0,90 & \\
\hline
\end{tabular}

M. cf. M. gallegosense

SC3645 $1,13 \quad 1,17 \quad 1,07 \quad 1,33$ 
Dichos ejemplares son muy similares y la diferencia más importante es que MACN Pv SC550 muestra un metacónido algo más proyectado en sentido anterior. Otras diferencias son aparentes y producto de distintos estados de desgaste y de conservación.

Hasta el momento se conocen molares superiores de sólo tres especies de microbiotéridos fósiles. En orden creciente de tamaño: Microbiotherium patagonicum, Microbiotherium tehuelchum y Pachybiotherium illuminatum (Marshall, 1982; Goin et al., 2010). A las dos primeras se han asignado restos que presentan la serie molar completa, mientras que de P. illuminatum sólo se conoce el M3 en muy mal estado de preservación. M. patagonicum, sumado a su pequeño tamaño, es de aspecto más grácil que las otras especies, con el protocono proporcionalmente más corto y la plataforma estilar algo más desarrollada. $M$. tehuelchum posee los molares proporcionalmente más largos, con los cónulos más marcados y un fuerte cíngulo mesial. Finalmente, Pachybiotherium illuminatum posee un tamaño considerablemente mayor que el de las dos especies anteriores, siendo cuatro veces mayor que los ejemplares de mayor tamaño de $M$. $p a-$ tagonicum y más de 3,5 veces mayor que $M$. tehuelchum. Además, el paracono y el metacono en P. illuminatum son de un tamańo relativo mayor que en las otras dos especies y la región estilar está aún más reducida.

El M2 MACN Pv SC3648, tentativamente referido aquí a $M$. divisum, presenta el protocono de gran tamaño, con el paracónulo y el metacónulo claramente visibles y un cíngulo mesial bien desarrollado. El paracono posee el ápice roto, pero debió ser más bajo que el metacono. La repisa estilar está rota en la región correspondiente a la $\mathrm{StC}$; sin embargo, se han preservado las cúspides $\mathrm{StA}, \mathrm{StB}$ y $\mathrm{StD}$, que se encuentran proporcionalmente más desarrolladas que en los M2 de M. tehuelchum y $M$. patagonicum. El gran desarrollo del protocono en el M2 sugiere que su antagonista oclusal poseía un talónido grande, como en $M$. divisum, más desarrollado que el de $M$. tehuelchum. El tamaño de este diente (Tab. 1) es mucho menor (menos de un tercio) que el del M3 asignado por Goin et al. (2010) a Pachybiotherium illuminatum, semejante al de M. tehuelchum y algo mayor que el de $M$. patagonicum. Sin embargo, aplicando el cociente $\mathrm{M} 2 / \mathrm{m} 2$ propuesto por Clemens (1966) para asociar molares superiores en inferiores, su tamańo resulta equivalente al del m2 MACN Pv SC520 asignado aquí a M. divisum (M2/ $\mathrm{m} 2=0,98)$. Esta asignación debe considerarse tentativa y sólo podrá ser corroborada con futuros hallazgos de molares superiores e inferiores asociados.
Microbiotherium cf. M. gallegosense Sinclair, 1906

\section{Figura 3.5}

Holotipo de M. gallegosense. AMNH 9591, un fragmento de mandíbula derecha, con $\mathrm{p} 3-\mathrm{m} 2$ completos, procedente de la Formación Santa Cruz aflorante en el margen norte del río Gallegos, en las cercanías de la Estancia Felton.

Material referido. MACN Pv SC3644, un fragmento de dentario izquierdo con el m1; MACN Pv SC3645, un m1 derecho aislado (Fig. 3.5).

Procedencia geográfica y estratigráfica. Ambos ejemplares provienen de la secuencia media de la Formación Pinturas en Portezuelo Sumich Sur.

Observaciones. Microbiotherium gallegosense es la especie más grande del género y sólo es conocida hasta el momento en niveles de edad santacrucense de la Formación Santa Cruz (Sinclair, 1906).

Los $\mathrm{m} 1$ aquí reportados se asemejan a los de $M$. gallegosense por ser más grandes que los molares asignados a las otras especies (Tab. 1), poseer un paracónido bien desarrollado, tener el entocónido proporcionalmente grande y el hipoconúlido, también grande, en una posición más labial que en las otras especies de Microbiotherium. Sin embargo, su tamaño es algo menor que el de $M$. gallegosense y el hipoconúlido es proporcionalmente más grande y está más proyectado distalmente. Si bien es claro que la similitud de estos ejemplares es mayor con $M$. gallegosense que con cualquier otra especie conocida, las diferencias observadas podrían indicar su pertenencia a una nueva especie.

\section{MicrobiotheriIdae indet.}

Figura 3.6

Material referido. MACN Pv SC3648C, un ?M2 izquierdo aislado.

Procedencia geográfica y estratigráfica. Secuencia media de la Formación Pinturas en Portezuelo Sumich Sur.

Medidas. Largo: 1,6 mm; Ancho: 1,72 mm (las medidas son estimativas).

Observaciones. El espécimen MACN Pv SC3648C posee un protocono de gran tamaño, la centrocrista recta, un metacónulo marcado y un pobre desarrollo de la región estilar. Todos estos caracteres permiten asignar este probable M2 a la Familia Microbiotheriidae. El diente está incompleto en su esquina posterolabial y presenta un alto grado de desgaste, por lo que resulta imposible efectuar una asignación taxonómica más precisa. Sin embargo, puede observarse que su tamaño habría sido algo menor que el M2 asignado más arriba a $M$. divisum. 


\section{DISCUSIÓN Y CONCLUSIONES}

La "fauna Astrapotericulense" fue reconocida por Florentino Ameghino (1900-1902) sobre la base de una pequeña colección de mamíferos fósiles obtenida por Carlos Ameghino, durante su noveno viaje a la Patagonia, en depósitos continentales expuestos en el valle superior del río Pinturas, noroeste de Santa Cruz. Si bien estos depósitos no pueden correlacionarse estratigráficamente con aquellos de la Formación Santa Cruz portadores de fauna santacrucense, Ameghino (1900-1902, 1906) interpretó que la asociación de mamíferos "Astrapotericulense" estaba constituida por elementos faunísticos distintivos, cuyo "estado evolutivo" indicaba que los depósitos portadores — sus "Couches a Astrapothericulus" - debían ser más antiguos. Esta idea fue seguida por Castellanos (1937), quien acuñó el término "Pinturense" para referirse al "Astrapotericulense" de Ameghino. Sin embargo, en base a los listados faunísticos provistos por Ameghino (1900-1902, 1906), Wood y Patterson (1959) consideraron que la "fauna Astrapotericulense" no mostraba elementos suficientemente distintivos como para ser considerada más que una mera fauna local santacrucense.

Las colecciones más recientes efectuadas en sedimentos de la Formación Pinturas, presuntamente equivalentes a las "Couches à Astrapothericulus", aportaron una gran cantidad de restos de vertebrados fósiles (especialmente mamíferos) con detallada información sobre su posición estratigráfica (Bown y Larriestra, 1990; Bown y Fleagle, 1993), permitiendo poner a prueba la propuesta original de Ameghino. Los estudios bioestratigráficos basados en roedores caviomorfos y litopternos (Kramarz y Bellosi, 2005; Kramarz y Bond, 2005) concluyeron que las secuencias inferior y media de la Formación Pinturas son portadoras de una asociación diferenciable y de rasgos más primitivos que sus contrapartes registradas en exposiciones de la Formación Santa Cruz en las localidades costeras con fauna típicamente santacrucense. Por el contrario, los niveles asignados a la secuencia superior de la Formación Pinturas son portadores de roedores y litopternos más derivados y en su mayoría también presentes en la fauna santacrucense (Kramarz y Bellosi, 2005; Kramarz y Bond, 2005). Concordantemente, las dataciones ${ }^{40} \mathrm{Ar} /{ }^{19} \mathrm{Ar}$ (Fleagle et al., 1995) indican que los niveles fosilíferos de las secuencias inferior y media de la Formación Pinturas son más antiguos que 16,5 Ma, mientras que la mayoría de los depósitos de la Formación Santa Cruz portadores de fauna santacrucense en Monte León y Monte Observación son más jóvenes que 16,5 Ma. Finalmente, Kramarz et al. (2010) concluyeron que las asociaciones provenientes de las secuencias inferior y media de la Formación Pinturas ("Astra- potericulense") y de los términos superiores de la Formación Sarmiento en la Barranca Sur del Lago Colhué Huapi (Upper Fossil Zone, Ré et al., 2010) corresponderían a una única unidad biocronológica que se extendería desde ca. 18,75 Ma a 16,5 Ma. Esta unidad, informalmente denominada "Pinturense", merece ser reconocida como una Edad Mamífero postcolhuehuapense-presantacrucense o bien como una subedad temprana dentro de la Edad Mamífero Santacrucense, representando un lapso más temprano que el Santacrucense en el sentido estricto (Kramarz et al., 2010).

El conjunto de roedores y litopternos que permiten tipificar la "fauna Astrapotericulense" está caracterizado por: (1) la presencia de taxones conocidos exclusivamente para este intervalo estratigráfico, incluyendo los taxones listados por Ameghino (1900-1902) como característicos de esta fauna [(e.g., Luantus propheticus Ameghino, 1899, Steiromys annectens (Ameghino, 1899), Tetramerorbinus prosistens (Ameghino, 1899)] y otros recientemente descriptos (e.g., Prostichomys bowni Kramarz, 2001, Perimys intemedius Kramarz, 2002, Picturotherium migueli Kramarz y Bond, 2005, Tetramerorhinus fleaglei Soria, 2001); (2) la presencia de taxones previamente conocidos para la Edad Mamífero Santacrucense (e.g., Neoreomys Ameghino, 1887); y (3) la presencia de taxones conocidos para la Edad Mamífero Colhuehuapense [e.g., Branisamyopsis Candela, 2003, Lambdaconus (Ameghino, 1897)] (Kramarz y Bellosi, 2005; Kramarz y Bond, 2005).

Todos los restos de marsupiales microbiotéridos aquí descriptos provienen de distintos niveles correspondientes a la secuencia media de la Formación Pinturas. Estos restos son asignables a: (1) Microbiotherium tehuelchum, previamente reconocida para la fauna típicamente santacrucense; (2) una especie de Microbiotherium con claras afinidades con M. gallegosense, también previamente reconocida para la Edad Santacrucense; y (3) Microbiotherium divisum, registrada con anterioridad en rocas de la Edad Colhuehuapense. A esto debe sumarse el registro de Pachybiotherium illuminatum descripta por Goin et al. (2010), también proveniente de la secuencia media de la Formación Pinturas (Portezuelo Sumich Sur) y sólo conocida hasta ahora para estos niveles. Esta composición claramente reproduce el patrón observado para los litopternos y los roedores caviomorfos, constituyendo un elemento adicional que sustenta la posición cronológica postcolhuehuapense-presantacrucense del "Pinturense".

A pesar de su extenso biocrón y su amplia distribución geográfica, los microbiotéridos constituyeron un grupo de relativamente poca abundancia y diversidad (e.g., Hershkovitz, 1999), al menos en comparación con otros micromarsupia- 
les sudamericanos (e.g., didelfimorfios, paucituberculados). La mayor diversidad de la familia se restringe al Mioceno temprano, con dos especies conocidas en estratos de edad colhuehuapense y unas cinco especies en estratos de edad santacrucense (sensu stricto) (Ameghino, 1887, 1902; Marshall, 1982; Hershkovitz, 1999). Sin embargo, no existía certeza de la coexistencia estricta de más de una especie en un único nivel estratigráfico. Las cuatro especies conocidas para la Formación Pinturas se registran en el nivel fosilífero representado en Portezulo Sumich Sur, constituyendo la mayor diversidad conocida de microbiotéridos asociados a un mismo nivel estratigráfico.

Sobre la base de su tamaño, la morfología de los molares y de la mandíbula, Goin et al. (2010) infirieron para Pachybiotherium illuminatum una alimentación predominantemente frugívora. Las especies de Microbiotherium se asemejan más en estos rasgos a Dromiciops gliroides, que posee una dieta omnívora de variación estacional, con alimentación preponderante insectívora-animalívora para algunos autores (e.g., Marshall, 1982; Hershkovitz, 1999; Goin et al., 2010) y con un componente frugívoro mayor para otros (e.g., Amico et al., 2009), mientras que Pachybiotherium illuminatum parece haber tenido una dieta con un componente frugívoro aún mayor, evidenciado por el gran tamańo relativo de la mandíbula, el robusto protocono de sus molares y la reducción en las estructuras de corte de los mismos (Goin et al., 2010). Las tres especies de Microbiotherium representadas en la secuencia media de la Formación Pinturas no presentan diferencias importantes entre sí en términos de tamaño, morfología de los molares y de la mandíbula. Sin embargo, Kay y Hylander (1978) y Dumont et al. (2000) señalaron que pequeñas diferencias en el tamaño corporal o en la morfología dentaria entre especies de micromamíferos estrechamente relacionadas pueden reflejar diferencias importantes en la dieta, por lo que la posible coexistencia de las tres especies identificadas de $\mathrm{Mi}$ crobiotherium no implicaría necesariamente una superposición de sus hábitos alimenticios.

\section{AGRADECIMIENTOS}

Agradecemos a M. Reguero por permitirnos estudiar materiales de la colección del MLP y a F. Tricárico (MACN) por las fotos tomadas con el Microscopio Electrónico de Barrido. A los revisores J. Babot y G. Martin por sus valiosos comentarios. L.C. agradece al CONICET (PIP 0361) y a la SECyT (PICT 2007-417).

\section{BIBLIOGRAFÍA}

Ameghino, F. 1887. Enumeración sistemática de las especies de mamíferos fósiles coleccionados por Carlos Ameghino en los terrenos eocenos de la Patagonia austral y depositados en el Museo de La Plata. Boletín del Museo de La Plata 1: 1-26.

Ameghino, F. 1891. Nuevos restos de mamíferos fósiles descubiertos por Carlos Ameghino en el Eoceno inferior de la Patagonia austral. Especies nuevas, adiciones y correcciones. Revista Argentina de Historia Natural 1: $289-328$

Ameghino, F. 1897. Mammifères crétacés de l'Argentine. Deuxième contribution à la connaissance de la faune mammalogique de couches à Pyrotherium. Boletín Instituto Geográfico Argentino 18: 406-521.

Ameghino, F. 1898. Sinopsis geológico-paleontológica. Segundo censo de la República Argentina. Folia, Buenos Aires 1: 112-255.

Ameghino, F. 1899. Sinopsis geológico-paleontológica. Suplemento (Adiciones y Correcciones), La Plata: 1-13.

Ameghino, F. 1900-1902. L'âge des formations sédimentaires de Patagonie. Anales de la Sociedad Cientifica Argentina 50: 109-131, 145-165, 209-229; 51: 20-39, 65-91; 52: 198-197, 244-250; 54: 161-180, 220-240, 283-342.

Ameghino, F. 1902. Première contribution à la connaissance de la faune mammalogique des couches à Colpodon. Boletín de la Academia Nacional de Ciencias de Córdoba 17: 71-138.

Ameghino, F. 1906. Les formations sédimentaires du Crétacé supérieur et du Tertiaire de Patagonie. Anales del Museo Nacional de Buenos Aires 8: 1-568.

Asher, R.J., Horovitz, I. y Sánchez-Villagra, M.R. 2004. First combined cladistic analysis of marsupial mammal interrelationships. Molecular Phylogenetics and Evolution 33: 240-250.

Amico, G.C., Rodríguez-Cabal, M.A. y Aizen, M.A. 2009. The potential key seed-dispersing role of the arboreal marsupial Dromiciops gliroides. Acta oecologica 35: 8-13.

Barrio, R.E. de, Scillato Yané, G. y Bond, M. 1984. La Formación Santa Cruz en el borde occidental del macizo del Deseado (provincia de Santa Cruz) y su contenido paleontológico. Noveno Congreso Geológico Argentino, Actas 4: 539-556.

Bown, T.M., Larriestra, C.N. y Powers, D.W. 1988. Análisis paleoambiental de la Formación Pinturas (Mioceno Inferior), Provincia de Santa Cruz. $2^{a}$ Reunión Argentina de Sedimentología (Buenos Aires), Actas 1: 31-35.

Bown, T.M. y Fleagle, J.G. 1993. Systematics, bioestratigraphy and dental evolution of the Palaeothentidae, Late Oligocene to Early-Middle Miocene (Deseadan-Santacrucian) Caenolestoid marsupials of South America. Paleontological Society, Memoir 29: 1-76.

Bown, T.M. y Laza, J. 1990. A fossil nest of a Miocene termite from southern Patagonia, Argentina, and the oldest record of the termites in South America. Ichnos 1: 73-79.

Bown, T.M. y Larriestra, C.N. 1990. Sedimentary paleoenvironments of fossil platyrrhine localities, Miocene Pinturas Formation, Santa Cruz province, Argentina. Journal of Human Evolution 19: 87-119.

Candela, A.M. 2003. A new porcupine (Rodentia, Hystricognathi, Erethizontidae) from the Early-Middle Miocene of Patagonia. Ameghiniana 39: 483-494.

Castellanos, A. 1937. Ameghino y la antigüedad del hombre sudamericano. Asociación Cultural de Conferencias de Rosario, Ciclo de Carácter General 2: 47-192.

Clemens, W.A. 1966. Fossil mammals of the Type Lance Formation, Wyoming. Part II. Marsupialia. University of California Publications in Geological Sciences 62: 1-122.

Dumont, E.R., Strait, S.G. y Friscia, A.R. 2000. Abderitid marsupials from the Miocene of Patagonia: an assessment of form, function, and evolution. Journal of Paleontology 74: 1161-1172.

Fleagle, J.G. 1990. New fossil platyrrhine from the Pinturas formation, southern Argentina. Journal of Human Evolution 19: 61-85.

Fleagle, J.G., Bown, T.M., Swisher, C. y Buckley, G. 1995. Age of the Pin- 
turas and Santa Cruz formations. $6^{\circ}$ Congreso Argentino de Paleontología y Bioestratigrafia (Trelew), Actas: 129-135.

Genise, J.F. y Bown, T.M. 1994. New Miocene scarabeid and hymenopterous nest and Early Miocene (Santacrucian) paleoenvironments, Patagonian Argentina. Ichnos 3: 107-117.

Goin, F.J. 1995. Los Marsupiales. En: M.A. Alberdi, G. Leone y E.P. Tonni (Eds.), Evolución biológica y climática de la Región Pampeana durante los últimos cinco millones de años. Un ensayo de correlación con el Mediterráneo occidental. Monografías del Museo Nacional de Ciencias Naturales, CSIC, Madrid, p. 165-179.

Goin, F.J., Woodburne, M.O., Case, J., Vizcaíno, S.F. y Reguero, M. 1999. New discoveries of "opossum-like" marsupials from Antarctica (Seymour Island, Middle Eocene). Journal of Mammalian Evolution 6: 335-365.

Goin, F.J, Tejedor, M.F., Abello, M.A. y Martin, G.M. 2010. Un nuevo microbiotérido (Mammalia, Marsupialia, Microbiotheria) de la Formación Pinturas (Mioceno temprano) de la provincia de Santa Cruz. Ameghiniana 47: 117-122.

González, L.R., Tejedor, M.F. y Scillato-Yané, G.J. 2006. Los Dasypodidae de la Formación Pinturas (Mioceno inferior), Provincia de Santa Cruz. Ameghiniana 43, Suplemento: 40R.

Hershkovitz, P. 1999. Dromiciops gliroides Thomas, 1894, last of the Microbiotheria (Marsupialia), with a review of the Family Microbiotheriidae. Fieldiana: Zoology, New Series 93: 1-60.

Kay, R.F. y Hylander, W.L. 1978. The dental structure of mammalian folivores with special reference to Primates and Phalangeroidea (Marsupialia). En: G. Montgomery (Ed.), The Ecology of Arboreal Folivores. Smithsonian Institution Press, Washington, p. 173-191.

Kramarz, A.G. 2001. Prostichomys bowni, un nuevo roedor Adelphomyinae (Hystricognathi, Echimyidae) del Mioceno medio-inferior de Patagonia, Argentina. Ameghiniana 38: 163-168.

Kramarz, A.G. 2002. Roedores chinchilloideos (Hystricognathi) de la Formación Pinturas, Mioceno temprano-medio de la provincia de Santa Cruz, Argentina. Revista del Museo Argentino de Ciencias Naturales, n.s. 4: $167-180$.

Kramarz, A.G. y Bellosi, E.S. 2005. Hystricognath rodents from the Pinturas Formation, Early- Middle Miocene of Patagonia, biostratigraphic and paleoenvironmental implications. Journal of South American Earth Sciences 18: 199-212.

Kramarz, A.G. y Bond, M. 2005. Los Astrapotheriidae (Mammalia) de la Formación Cerro Bandera, Mioceno temprano de Patagonia septentrional. Ameghiniana 42, Suplemento: 72R-73R.

Kramarz, A.G., Vucetich, M.G., Carlini, A.A., Ciancio, M.R., Abello, M.A., Deschamps, C.M. y Gelfo, J.N. 2010. A new mammal fauna at the top of the Gran Barranca sequence and its biochronological significance. En: R.H. Madden, A.A. Carlini, M.G. Vucetich y R.F. Kay (Eds.), The Paleontology of Gran Barranca: Evolution and Environmental Change through the Middle Cenozoic of Patagonia. Cambridge University Press, Cambridge, p. 264-277.

Marshall, L.G. 1982. Systematics of the South American marsupial family Microbiotheriidae. Fieldiana: Geology, New Series 10: 1-75.

Marshall, L. 1990. Fossil Marsupialia from the type Friasian Land Mammal Age (Miocene), Alto Río Cisnes, Aisén, Chile. Revista Geológica de Chile 17: 19-55.

Marshall, L., Hoffstetter, R. y Pascual, R. 1983. Mammals and Stratigraphy: geochronology of the continental mammal-bearing Tertiary of South America. Palaeovertebrata, Mémoire Extraordinaire: 1-93.

Marshall, L. y de Muizon, C. 1988. The dawn of the age of mammals in South America. National Geographic Research 4: 23-55.

Marshall, L., Pascual, R., Curtis, G.H. y Drake, R.E. 1977. South American geochronology-radiometric time scale for middle to late Tertiary mammal-bearing horizons in Patagonia. Science 195: 1325-1328.
Martin, G. 2003. Nuevas localidades para marsupiales patagónicos (Didelphimorphia y Microbiotheria) en el noroeste de la Provincia del Chubut, Argentina. Mastozoología Neotropical 10: 148-153.

Martin, G. 2010. Geographic distribution and historical occurrence of Dromiciops gliroides Thomas (Metatheria: Microbiotheria). Journal of Mammalogy 91: 1025-1035.

Nilsson, M.A., Churakov, G., Sommer, M., Van Tran, N., Zemann, A., Brosius, J. y Schmitz, J. 2010. Tracking marsupial evolution using archaic genomic retroposon insertions. PLoS Biology 8: 1-9.

Olson, D.M., Dinerstein, E., Wikramanayake, E.D., Burgess, N.D., Powell, G.V.N., Underwood, E.C., D’amico, J.A., Itoua, I., Strand, H.E., Morrison, J.C., Loucks, C.J., Allnutt, T.F., Ricketts, T.H., Kura, Y., Lamoreux, J.F., Wettengel, W.W., Hedao, P. y Kassem, K.R. 2001. Terrestrial ecoregions of the world: a new map of life on Earth. Bio Science 51: 933-938.

Palma, R.E. y Spotorno, A.E. 1999. Molecular systematics of marsupials based on the rRNA $12 S$ mitochondrial gene: the phylogeny of Didelphimorphia and of the living fossil Microbiotheriid Dromiciops gliroides Thomas. Molecular Phylogenetics and Evolution 13: 525-535.

Pascual, R. y Odreman Rivas, O.E. 1971. Evolución de las comunidades de los vertebrados del Terciario argentino. Los aspectos paleozoogeográficos y paleoclimáticos relacionados. Ameghiniana 8: 372-412.

Pascual, R., Ortega Hinojosa, E.J., Gondar, D. y Tonni, E. 1965. Las edades del Cenozoico mamalífero de la Argentina, con especial atención a aquellas del territorio bonaerense. Anales de la Comisión de Investigaciones Cientificas de la Provincia de Buenos Aires 6: 165-193.

Rae, T.C., Bown, T.M. y Fleagle, J.G. 1996. New Palaeothentid Marsupials (Caenolestoidea) from the Early Miocene of Patagonian Argentina. American Museum Novitates 3165: 1-10.

Ré, G.H., Bellosi, E.S., Heizler, M., Vilas, J.F., Madden, R.H., Carlini, A., Kay, R.F. y Vucetich, M.G. 2010. A geochronology for the Sarmiento Formation at Gran Barranca. En: R.H. Madden, A.A. Carlini, M.G. Vucetich y R.F. Kay (Eds.), The Paleontology of Gran Barranca: Evolution and Environmental Change through the Middle Cenozoic of Patagonia. Cambridge University Press, Cambridge, p. 46-58.

Sinclair, W.J. 1906. Marsupials of the Santa Cruz beds. Princeton University Expeditions to Patagonia 4: 330-460.

Simpson, G.G. 1940. Review of the mammal-bearing Tertiary of South America. Proceedings of the American Philosophical Society 83: 649-709.

Soria, M.F. (h). 2001. Los Proterotheriidae (Litopterna, Mammalia), sistemática, origen y filogenia. Monografías del Museo Argentino de Ciencias Naturales 1: 1-167.

Szalay, F.S. 1982. A new appraisal of marsupial phylogeny and classification. En: M. Archer (Ed.), Carnivorous Marsupials. Royal Zoological Society of New South Wales, Sydney, p. 612-640.

Thomas, O. 1894. On Micoureus griseus, Desm., with the description of a new genus and species of Didelphyidae. Annals and Magazine of Natural History 6: 184-188.

Wood, A.E. y Patterson, B. 1959. The rodents of the Deseadan Oligocene of Patagonia and the beginnings of South American rodent evolution. Bulletin of the Museum of Comparative Zoology 120: 281-428.

doi: 10.5710/AMGH.22.2.2012.524

Recibido: 3 de agosto de 2011

Aceptado: 22 de febrero de 2012 